TITLE:

\title{
MONOTONE CONVOLUTION AND MONOTONE INFINITE DIVISIBILITY FROM COMPLEX ANALYTIC VIEWPOINT
}

\author{
$\operatorname{AUTHOR}(\mathrm{S}):$ \\ HASEBE, TAKAHIRO
}

CITATION:

HASEBE, TAKAHIRO. MONOTONE CONVOLUTION AND MONOTONE INFINITE DIVISIBILITY FROM COMPLEX ANALYTIC VIEWPOINT. Infinite Dimensional Analysis, Quantum

Probability and Related Topics 2010, 13(01): 111-131

\section{ISSUE DATE:}

2010

URL:

http://hdl.handle.net/2433/128874

\section{RIGHT:}

(C) 2010 World Scientific Publishing Company; This is not the published version. Please cite only the published version.; この論文は出版社版で ありません。引用の際には出版社版をご確認ご利用ください。 


\title{
Monotone Convolution and Monotone Infinite Divisibility from Complex Analytic Viewpoint
}

\author{
Takahiro Hasebe \\ JSPS Research Fellow (DC), Graduate School of Science, \\ Kyoto University, Kyoto 606-8502, Japan \\ E-mail: hsb@kurims.kyoto-u.ac.jp
}

\section{Introduction}

Muraki has defined the notion of monotone independence in the preprint [14]. Then monotone convolution has been defined by the probability distribution of the sum of two non-commutative random variables which are monotone independent. He also clarified the following important points: an algebraic construction of monotone product of operator algebras; a complex analytic method in monotone convolution. In this paper, we focus on the complex analytic method.

In the complex analytic method, the reciprocal Cauchy transform

$$
H_{\mu}(z):=\frac{1}{G_{\mu}(z)}
$$

is important, where

$$
G_{\mu}(z):=\int_{\mathbb{R}} \frac{1}{z-x} d \mu(x)
$$

is the Cauchy transform of a probability measure $\mu$. Muraki has proved that the monotone convolution $\mu \triangleright \nu$ of two probability measures $\mu$ and $\nu$ with compact supports is characterized by the relation

$$
H_{\mu \triangleright \nu}(z)=H_{\mu}\left(H_{\nu}(z)\right) .
$$

This relation naturally allows us to extend monotone convolution to probability measures with unbounded supports. (Recently, probability distributions with unbounded supports have been discussed in [8] from an operator theoretic point of view.)

Similarly to the classical case, Muraki has defined the notion of monotone infinitely divisible distributions (often denoted by $\triangleright$-infinitely divisible distributions). Muraki has proved that when probability measures are compactly supported, there is a natural one-to-one correspondence among a $\triangleright$-infinitely divisible probability measure, a weakly continuous one-parameter monotone convolution semigroup of probability measures, and a vector field in the upper half plane [14]. The complete correspondence has been proved by Belinschi [3]. Now we state the result.

Theorem 1.1 [3, 14] There is a one-to-one correspondence among the following four objects:

(1) a $\triangleright$-infinitely divisible distribution $\mu$; 
(2) a weakly continuous $\triangleright$-convolution semigroup $\left\{\mu_{t}\right\}_{t \geq 0}$ with $\mu_{0}=\delta_{0}$ and $\mu_{1}=\mu$;

(3) a composition semigroup of reciprocal Cauchy transforms $\left\{H_{t}\right\}_{t \geq 0}$ with $H_{0}=$ id and $H_{1}=$ $H_{\mu}$

(4) a vector field on the upper half plane of the form

$$
A(z)=-\gamma+\int_{\mathbb{R}} \frac{1+x z}{x-z} d \tau(x)
$$

where $\gamma \in \mathbb{R}$ and $\tau$ is a positive finite measure.

The vector field $A$, which we call the associated vector field to a $\triangleright$-infinitely divisible distribution, is obtained from (3) by

$$
A(z)=\lim _{t \searrow 0} \frac{H_{t}(z)-z}{t} .
$$

Conversely, $\left\{H_{t}\right\}$ is obtained from the following ordinary differential equation:

$$
\begin{aligned}
& \frac{d}{d t} H_{t}(z)=A\left(H_{t}(z)\right), \\
& H_{0}(z)=z,
\end{aligned}
$$

for $z \in \mathbb{C} \backslash \mathbb{R}$.

The fact that the solution of (1.6) does not explode in finite time has been proved in [4]. This vector field is not necessarily complete (we show in Section 2 that a vector field associated to a one-parameter monotone convolution semigroup $\left\{\mu_{t}\right\}_{t \geq 0}$ is complete if and only if $\mu_{t}=\delta_{t a}$ for some $a \in \mathbb{R}$.)

In Section 2, we study the injectivity of the reciprocal Cauchy transforms of $\triangleright$-finitely divisible and $\triangleright$-infinitely divisible distributions. The motivation of the study of finitely divisible distributions is as follows. The notion of $\triangleright$-divisibility and the problem of finding a vector field have a connection to the problem in infinite dimensional Lie group theory: "does a diffeomorphism have a form $\exp (X)$ for some vector field $X$ ?" The answer is negative: it is known that there is a diffeomorphism $\phi$ on any manifold which cannot have an expression of $\phi=\exp (X)$ where $X$ is a vector field. A concrete example of such a diffeomorphism is $\phi(\theta)=\theta+\frac{\pi}{n}+\epsilon \sin ^{2}(n \theta)$ on $S^{1}$ for a sufficiently large integer $n$ and a real number $0<\epsilon<\frac{1}{n}$ [11]. Actually, $\phi$ is proved to have no square root, i.e., there exists no diffeomorphism $g$ such that $\phi=g \circ g$. When we treat injective mappings in monotone probability theory, this example is instructive since it implies the importance of the notion of " $n$-divisibility." Therefore, we define in Section 2 the notion of $n$-divisibility of a probability measure and discuss its connection to injectivity of the reciprocal Cauchy transform.

In Section 3, we show an interlacing property of the monotone convolution of atomic measures (Theorem 3.1) and then we conclude that the monotone convolution of atomic measures with $m$ and $n$ atoms contains just $m n$ atoms (Corollary 3.3). In addition, motivated by the study in Section 2 , we clarify that the existence of an atom in a $\triangleright$-infinitely divisible distribution puts a restriction on the distribution (Theorem 3.5).

In Section 4 we classify strictly $\triangleright$-stable distributions (or equivalently, $\triangleright$-infinitely divisible and self-similar distributions). The result is very similar to the free and boolean cases. 
In Section 5, several examples of $\triangleright$-infinitely divisible distributions are shown, which are useful in understanding of the results in this paper.

This paper contains several results of properties of monotone convolution based on the complex analytic method. Among them, the following two theorems are most important results.

Theorem 3.5 If a $\triangleright$-infinitely divisible distribution $\nu$ contains an isolated atom at $a, \nu$ is of the form $\nu=\nu(\{a\}) \delta_{a}+\nu_{a c}$, where $\nu_{a c}$ is absolutely continuous w.r.t. the Lebesgue measure and $a \notin \operatorname{supp} \nu_{a c}$. Moreover, we have

$$
\left\{u \in \operatorname{supp} \nu \backslash\{a\} ; \limsup _{v \searrow 0}\left|G_{\nu}(u+i v)\right|=\infty\right\}=\emptyset .
$$

A strictly $\triangleright$-stable distribution is a $\triangleright$-infinitely divisible distribution characterized by the self-similarity. Let $\left\{\mu_{t}\right\}_{t \geq 0}$ be a weakly continuous monotone convolution semigroup with $\mu_{0}=$ $\delta_{0}$. Then $\mu:=\mu_{1}$ is said to be strictly $\triangleright$-stable if for any $a>0$, there exists $b(a)>0$ such that

$$
\mu_{a}=D_{b(a)} \mu
$$

where $D_{\lambda}$ is the dilation operator defined by $D_{\lambda} \mu(B)=\mu\left(\lambda^{-1} B\right)$. We show that there exists a unique real number $\alpha$ for a given nontrivial strictly $\triangleright$-stable distribution $\mu$ such that $b(a)=a^{\frac{1}{\alpha}}$ for all $a>0$. This real number $\alpha$ is called the index of $\mu$.

Define a semigroup of probability distributions $\left\{\mu_{t}^{(\alpha, b, 0)}\right\}_{t \geq 0}$ characterized by the vector field $A^{(\alpha, b, 0)}(z)=\frac{b}{\alpha} z^{1-\alpha}$.

Theorem 4.5 Assume that $\mu$ is a strictly $\triangleright$-stable distribution with $\mu \neq \delta_{0}$. Then the index $\alpha$ of $\mu$ satisfies $0<\alpha \leq 2$. Moreover, there exists $b \in \mathbb{C}$ such that $\mu=\mu_{1}^{(\alpha, b, 0)}$, where $b$ satisfies the following conditions:

$$
\begin{aligned}
& 0 \leq \arg b \leq \alpha \pi \text { if } 0<\alpha \leq 1, \\
& \cdot(\alpha-1) \pi \leq \arg b \leq \pi \text { if } 1<\alpha \leq 2 .
\end{aligned}
$$

\section{Injectivity of Reciprocal Cauchy Transform}

For a $\triangleright$-infinitely divisible distribution $\mu$, the injectivity of $H_{\mu}$ follows from the uniqueness of the solution of the ordinary differential equation (1.6). This injectivity can be seen as the counterpart of the classical fact that for any infinitely divisible distribution, its Fourier transform has no zero point on $\mathbb{R}$. The result in [3] implies that $H_{\mu}$ is injective for any $\triangleright$-infinitely divisible distribution $\mu$ (the support of which may be unbounded). If a probability distribution is of finite variance, however, the injectivity property can be shown in a way different from [3]. We do not need to embed a probability measure in a convolution semigroup. Moreover, the method is applicable to finitely divisible distributions. In this section we present the proof.

We denote by $H^{n}:=H \circ H \circ \cdots \circ H$ the $n$ fold composition of a map $H$ throughout this paper.

Define a set of probability measures $\Phi:=\left\{\mu ; H_{\mu}\right.$ is injective $\}$. We shall prove (b) and (c) of the following properties of the set $\Phi$ :

(a) $\mu, \nu \in \Phi \Longrightarrow \mu \triangleright \nu \in \Phi$; 
(b) $\Phi$ is closed under the weak topology of probability measures;

(c) If $\mu$ is a $\triangleright$-infinitely divisible distribution with finite variance, then $\mu \in \Phi$;

(c') If $\mu$ is a $\triangleright$-infinitely divisible distribution, then $\mu \in \Phi$.

The proof of (a) is simple. The assumption "finite variance" in $(c)$ is not needed if we use the result in [3], and hence, (c') holds. These results are contained in Theorem 2.4 and Proposition 2.9. The set $\Psi:=\{\mu ; \mu$ is $\triangleright$-infinitely divisible $\}$ is difficult to analyze except for probability measures with compact supports. For instance, properties (a) and (b) seem to be difficult to prove for $\Psi$. We have defined $\Phi$ for this reason and aim to analyze $\Phi$ instead of $\Psi$. (c) (or (c')) is useful as a criterion for $\triangleright$-infinite divisibility. An application of property (c') is in Theorem 3.5 .

In the classical case, it is known that

$$
\{\mu ; \mu \text { is infinitely divisible }\} \varsubsetneqq\{\mu ; \hat{\mu}(\xi) \neq 0 \text { for all } \xi \in \mathbb{R}\},
$$

where $\hat{\mu}(\xi):=\int e^{i x \xi} d \mu(x), \xi \in \mathbb{R}$. In order to construct an example of $\mu$ whose Fourier transform has no zero points but is not infinitely divisible, we need to make a function $f(\xi)$ such that $\exp (f(\xi))$ is positive definite and $\exp \left(\frac{1}{n} f(\xi)\right)$ is not positive definite for some $n \in \mathbb{N}$. Such an example is $\hat{\mu}(\xi)=\frac{1}{2}\left(e^{-\frac{\xi^{2}}{2}}+e^{-|\xi|}\right)$. This is a positive definite function and there exists a distribution $\mu$ by Bochner's theorem. The fact that the distribution is not infinitely divisible is shown by Corollary 9.9 in Chapter 4 of the book [20].

In an analogy with (2.1), the conjecture

$$
\{\mu ; \triangleright \text {-infinitely divisible }\} \varsubsetneqq\left\{\mu ; H_{\mu} \text { is injective }\right\}
$$

comes up in the monotone case. The author has not been able so far to prove this fact.

We prepare for the proof of (b) and (c). The next proposition is taken from [10] in a slightly more general version.

Proposition 2.1 [10] A probability measure $\mu$ has a finite variance $\sigma^{2}(\mu)$ if and only if $H_{\mu}$ has the representation

$$
H_{\mu}(z)=a+z+\int_{\mathbb{R}} \frac{1}{x-z} d \rho(x),
$$

where $a \in \mathbb{R}$ and $\rho$ is a positive finite measure. Furthermore, we have $\rho(\mathbb{R})=\sigma^{2}(\mu)$ and $a=-m(\mu)$, where $m(\mu)$ denotes the mean of $\mu$ and $\sigma^{2}(\mu)$ denotes the variance of $\mu$.

Definition 2.2 (1) A probability measure $\mu$ is said to be $\triangleright$ - $k$-divisible if there exists a probability measure $\mu_{k}$ such that $\mu=\mu_{k}^{\triangleright k}$.

(2) A probability measure $\mu$ is said to be $\triangleright$-infinitely divisible if for any integer $1 \leq k<\infty$, there exists a probability measure $\mu_{k}$ such that $\mu=\mu_{k}^{\triangleright k}$. We call $\mu_{k}$ a $k$-th root of $\mu$.

Let $\mu$ and $\nu$ be probability measures. For each $x \in \mathbb{R}$ let $\nu_{x}$ be a probability measure defined by the equation $[14]$

$$
H_{\nu_{x}}(z)=H_{\nu}(z)-x
$$


and we have the representation of a monotone convolution in the form $\mu \triangleright \nu(A)=\int_{\mathbb{R}} \nu_{x}(A) d \mu(x)$. It follows from this representation that monotone convolution is affine in the left component:

$$
\left(\theta_{1} \mu+\theta_{2} \nu\right) \triangleright \lambda=\theta_{1}(\mu \triangleright \lambda)+\theta_{2}(\nu \triangleright \lambda)
$$

for all probability measures $\mu, \nu$ and $\lambda$ and $\theta_{1}, \theta_{2} \geq 0, \theta_{1}+\theta_{2}=1$. It should be noted that $\mu_{x}$ is weakly continuous with respect to $x$. The reader is referred to Theorem 2.5 in [10] for the proof. The measurability of $\mu_{x}(A)$ for any Borel set $A$ (denoted as $A \in \mathcal{B}(\mathbb{R})$ ) follows from the weak continuity. In fact, for an open set $A$, the function $x \longmapsto \mu_{x}(A)$ is lower semicontinuous, and hence, is measurable. Define the set $\mathcal{F}:=\left\{A \in \mathcal{B}(\mathbb{R}) ; x \longmapsto \mu_{x}(A)\right.$ is measurable $\}$. Every open set is contained in $\mathcal{F}$ and $\mathcal{F}$ is a $\sigma$-algebra; therefore, $\mathcal{F}=\mathcal{B}(\mathbb{R})$.

The next lemma is almost the same as Lemma 6.3 in [14].

Lemma 2.3 Assume that a probability measure $\mu$ has finite variance and that $\mu$ is $\triangleright$-k-divisible. Then a $k$-th root $\mu_{k}$ of $\mu$ has finite variance. Therefore, $\mu_{k}$ has the integral representation in the form

$$
H_{\mu_{k}}(z)=a_{k}+z+\int_{\mathbb{R}} \frac{1}{x-z} d \rho_{k}(x) .
$$

Moreover, it holds that $a_{k}=\frac{1}{k} a$ and $\rho_{k}(\mathbb{R})=\frac{\rho(\mathbb{R})}{k}$, where $(a, \rho)$ is a pair which appears in the representation (2.3).

Proof. The monotone convolution $\mu=\mu_{k}^{\triangleright k}$ can be expressed as

$$
\mu(A)=\int_{\mathbb{R}} \mu_{k, x}(A) d \mu_{k}^{\triangleright k-1}(x) .
$$

Since $\mu$ has finite variance, we have

$$
\int_{\mathbb{R}} y^{2} d \mu(y)=\int_{\mathbb{R}} d \mu_{k}^{\triangleright k-1}(x) \int_{\mathbb{R}} y^{2} d \mu_{k, x}(y)<\infty .
$$

Hence there exists some $y_{0} \in \mathbb{R}$ such that $\sigma^{2}\left(\mu_{k, y_{0}}\right)<\infty$. By Proposition 2.1, we obtain the representation

$$
H_{\mu_{k, y_{0}}}(z)=b_{k}+z+\int_{\mathbb{R}} \frac{1}{x-z} d \rho_{k}(x),
$$

and the representation for $H_{\mu_{k}}$

$$
H_{\mu_{k}}(z)=b_{k}+y_{0}+z+\int_{\mathbb{R}} \frac{1}{x-z} d \rho_{k}(x) .
$$

Therefore, we have $\sigma^{2}\left(\mu_{k}\right)<\infty$ again by Proposition 2.1 . 
Next we have

$$
\begin{aligned}
H_{\mu}(z) & =H_{\mu_{k}}\left(H_{\mu_{k}}^{k-1}(z)\right) \\
& =a_{k}+H_{\mu_{k}}^{k-1}(z)+\int_{\mathbb{R}} \frac{1}{x-H_{\mu_{k}^{\triangleright k-1}}(z)} \rho_{k}(d x) \\
& =a_{k}+H_{\mu_{k}}^{k-1}(z)-\int_{\mathbb{R}} G_{\left(\mu_{k}^{\triangleright k-1}\right)_{x}} \rho_{k}(d x) \\
& =a_{k}+H_{\mu_{k}}^{k-1}(z)-\int_{\mathbb{R}} \rho_{k}(d x) \int_{\mathbb{R}} \frac{\left(\mu_{k}^{\triangleright k-1}\right)_{x}(d y)}{z-y} \\
& =a_{k}+H_{\mu_{k}}^{k-1}(z)+\int_{\mathbb{R}} \frac{1}{y-z} \int_{\mathbb{R}}\left(\mu_{k}^{\triangleright k-1}\right)_{x}(d y) \rho_{k}(d x) \\
& =a_{k}+a_{k}+H_{\mu_{k}}^{k-2}(z)+\int_{\mathbb{R}} \frac{1}{y-z}\left(\int_{\mathbb{R}}\left(\mu_{k}^{\triangleright k-1}\right)_{x}(d y) \rho_{k}(d x)+\int_{\mathbb{R}}\left(\mu_{k}^{\triangleright k-2}\right)_{x}(d y) \rho_{k}(d x)\right) \\
& =\cdots \\
& =k a_{k}+z+\int_{\mathbb{R}} \frac{1}{y-z}\left(\sum_{m=0}^{k-1} \int_{\mathbb{R}}\left(\mu_{k}^{\triangleright m}\right)_{x}(d y) \rho_{k}(d x)\right),
\end{aligned}
$$

where $\mu_{k}^{\triangleright 0}:=\delta_{0}$. ¿From the uniqueness of the representation, we obtain $a=k a_{k}$ and

$$
\rho(d y)=\sum_{m=0}^{k-1} \int_{\mathbb{R}}\left(\mu_{k}^{\triangleright m}\right)_{x}(d y) \rho_{k}(d x),
$$

Hence we have $\rho(\mathbb{R})=k \rho_{k}(\mathbb{R})$. q.e.d.

Theorem 2.4 Let $\mu$ be a probability measure with finite variance.

(1) Assume that $\mu$ is $\triangleright$-n-divisible. If $z_{1} \neq z_{2}$ are two points in $\mathbb{C}_{+}$satisfying $\operatorname{Im} z_{1} \cdot \operatorname{Im} z_{2}>\frac{\rho(\mathbb{R})}{n}$, then $H_{\mu}\left(z_{1}\right) \neq H_{\mu}\left(z_{2}\right)$. In particular, $H_{\mu}$ is injective in $\left\{z \in \mathbb{C}_{+} ; \operatorname{Im} z>\sqrt{\frac{\rho(\mathbb{R})}{n}}\right\}$. Moreover, the constant $\frac{\rho(\mathbb{R})}{n}$ is optimal.

(2) Assume that $\mu$ is $\triangleright$-infinitely divisible. Then $H_{\mu}\left(\right.$ and hence $\left.G_{\mu}\right)$ is injective.

Proof. (1) We use the same notation for the integral representation of $\mu$ and $\mu_{k}$ as the one adopted in the previous lemma. Pick an arbitrary real number $r<1$ and fix it. Let $z_{1}, z_{2}$ be any two points satisfying $\frac{\rho(\mathbb{R})}{n \operatorname{Im} z_{1} \operatorname{Im} z_{2}}<r$. 
First we have

$$
\begin{aligned}
\left|H_{\mu_{n}}\left(z_{1}\right)-H_{\mu_{n}}\left(z_{2}\right)\right| & =\left|z_{1}-z_{2}+\int_{\mathbb{R}}\left(\frac{1}{x-z_{1}}-\frac{1}{x-z_{2}}\right) d \rho_{n}(x)\right| \\
& \geq\left|z_{1}-z_{2}\right|-\left|\int_{\mathbb{R}} \frac{z_{2}-z_{1}}{\left(x-z_{1}\right)\left(x-z_{2}\right)} d \rho_{n}(x)\right| \\
& \geq\left|z_{1}-z_{2}\right|-\left|\int_{\mathbb{R}} \frac{\left|z_{2}-z_{1}\right|}{\operatorname{Im} z_{1} \operatorname{Im} z_{2}} d \rho_{n}(x)\right| \\
& \geq\left|z_{1}-z_{2}\right|(1-r) .
\end{aligned}
$$

Since $\operatorname{Im} H_{\mu_{n}}(z) \geq \operatorname{Im} z$ for all $z \in \mathbb{C}_{+}$, we can iterate the inequality:

$$
\begin{aligned}
\left|H_{\mu}\left(z_{1}\right)-H_{\mu}\left(z_{2}\right)\right| & =\left|H_{\mu_{n}}^{n}\left(z_{1}\right)-H_{\mu_{n}}^{n}\left(z_{2}\right)\right| \\
& \geq\left|z_{1}-z_{2}\right|(1-r)^{n} .
\end{aligned}
$$

Therefore, $z_{1} \neq z_{2}$ implies $H_{\mu}\left(z_{1}\right) \neq H_{\mu}\left(z_{2}\right)$ since $r$ can be taken arbitrary near to 1 .

The optimality of the constant $\frac{\rho(\mathbb{R})}{n}$ will be proved in Example 2.7 shown later.

(2) For any $z_{1}, z_{2} \in \mathbb{C}_{+}$we take $n$ large enough so that $\operatorname{Im} z_{1} \cdot \operatorname{Im} z_{2}>\frac{\rho(\mathbb{R})}{n}$, then we can use the result (1). q.e.d.

Example 2.5 $H_{\mu}$ (or $G_{\mu}$ ) of the following probability measures are all injective:

(1) Arcsine law $d \mu(x)=\frac{1}{\pi \sqrt{2-x^{2}}} 1_{(-\sqrt{2}, \sqrt{2})}(x) d x, H_{\mu}(z)=\sqrt{z^{2}-2}$,

(2) Uniform distribution $d \mu=\frac{1}{b-a} 1_{(a, b)}(x) d x, G_{\mu}(z)=\frac{1}{b-a} \log \left(\frac{z-a}{z-b}\right)$,

(3) Wigner's semicircle law $d \mu(x)=\frac{1}{2 \pi} \sqrt{4-x^{2}} 1_{(-2,2)}(x) d x, H_{\mu}(z)=\frac{z+\sqrt{z^{2}-4}}{2}$,

(4) Normal distribution $d \mu(x)=\frac{1}{\sqrt{2 \pi}} e^{-\frac{x^{2}}{2}} d x$.

The injectivity in the cases (1), (2) and (3) can be confirmed directly. To prove the injectivity of the Stieltjes transform of the normal distribution, we use a general criterion for injectivity proved by Aksent'ev, which is also applicable to (1), (2) and (3). The reader is referred to a survey article [2] for details.

Theorem 2.6 (Aksent'ev) Let $a<c<b$ and let $p:[a, b] \rightarrow[0, \infty)$ be a function which is not identically zero, does not decrease in the interval $(a, c)$ and does not increase in the interval $(c, b)$. Then the function $\int_{a}^{b} \frac{1}{z-x} p(x) d x$ is injective in $\mathbb{C} \backslash[a, b]$.

When we apply this theorem to the normal distribution $\mu$, first we restrict the distribution to the closed interval $[-n, n]$, which we denote by $\mu_{n}$, and then take the limit $n \rightarrow \infty$. By Theorem 
2.6, $G_{\mu_{n}}$ is injective in $\mathbb{C}_{+}$. Since $\mu_{n} \rightarrow \mu$ weakly, $G_{\mu}$ is injective in $\mathbb{C}_{+}$by Proposition 2.9 shown later.

Arcsine law is the only distribution known to be $\triangleright$-infinitely divisible in the above examples. It is an interesting question whether the other examples are $\triangleright$-infinitely divisible or not.

Example 2.7 Next we treat atomic measures. We define $\nu:=\lambda_{1} \delta_{a}+\lambda_{2} \delta_{b}$ with $\lambda_{1}+\lambda_{2}=1$ and $a \neq b$. Its Cauchy transform is

$$
\begin{aligned}
G_{\nu}(z) & =\frac{\lambda_{1}}{z-a}+\frac{\lambda_{2}}{z-b} \\
& =\frac{z-\left(\lambda_{2} a+\lambda_{1} b\right)}{(z-a)(z-b)} .
\end{aligned}
$$

For simplicity, we consider the case $b=-a, a>0$ and $\lambda_{1}=\lambda_{2}=\frac{1}{2}$. Then $H_{\nu}(z)=\frac{z^{2}-a^{2}}{z}$, $\sigma^{2}(\nu)=a^{2}, m(\nu)=0$. By Proposition 2.1, $\rho(\mathbb{R})=a^{2}$. Take $z_{1}=s i$ and $z_{2}=t i$ such that $s t=a^{2}$. For instance, take $z_{1}=\frac{a}{2} i$ and $z_{2}=2 a i$. Clearly we have $z_{1} \neq z_{2}$ and $\operatorname{Im} z_{1} \operatorname{Im} z_{2}=a^{2}$. Moreover, one can see that $H_{\nu}\left(z_{1}\right)=H_{\nu}\left(z_{2}\right)$. Therefore, $\nu$ is not 2-divisible by Theorem 2.4. Moreover, the optimality of the constant $\frac{\rho(\mathbb{R})}{n}$ is proved by the example $\nu^{\triangleright n}$. In fact, for any integer $n$, it holds that $\sigma^{2}\left(\nu^{\triangleright n}\right)=n a^{2}$ and $m\left(\nu^{\triangleright n}\right)=0$ by Lemma 2.3. If we take $z_{1}=\frac{a}{2} i$ and $z_{2}=2 a i$ again, then $H_{\nu \triangleright n}=H_{\nu}^{n}$ maps $z_{1}$ and $z_{2}$ to the same point. Hence the proof of Theorem 2.4 has been completed.

It is clear that $\nu^{\triangleright 2}$ is 2-divisible. In addition, it is not difficult to prove that $\nu^{\triangleright 2}$ is not 3-divisible in application of Theorem 2.4.

We have seen the divisibility of atomic measures through an example. There is a question whether $H_{\nu}$ for $\nu=\sum_{k=1}^{m} \lambda_{k} \delta_{a_{k}}$ is $\triangleright$-infinitely divisible or not. The answer is given in Section 3 , Theorem 3.5.

In the classical probability theory, the set of infinitely divisible distributions is closed under the weak topology [18]. In monotone probability theory, however, this is difficult to prove and the proof is unknown. Instead we show that the injectivity property is conserved under the weak topology. The proof of the next Lemma is the analogy of the case of characteristic functions, but the tightness of probability measures is not needed. Hence we can give a proof without Prohorov's theorem.

Lemma 2.8 If a sequence of positive finite measures $\left\{\mu_{n}\right\}$ converges weakly to a positive finite measure $\mu$, then the Cauchy transform $G_{\mu_{n}}$ converges to $G_{\mu}$ locally uniformly on $\mathbb{C}_{+}$.

Proof. Pointwise convergence follows from the definition of the weak convergence of $\left\{\nu_{n}\right\}$. Locally uniform convergence is a consequence of Montel's theorem. q.e.d.

Proposition 2.9 Let $\left\{\mu_{n}\right\}$ be a sequence of positive finite measures whose $G_{\mu_{n}}$ are injective. If $\mu_{n}$ converges weakly to a nonzero positive finite measure $\mu$, then $G_{\mu}$ is injective.

Proof. This fact comes from Lemma 2.8 and the fact that the set of injective analytic functions on a domain is closed under the locally uniform topology (see Section 6 of Chapter 9 in [15]). Then the limit function is also injective on the domain. q.e.d. 
After we stated some properties about the injectivity of $H_{\mu}$, it is natural to ask when $H_{\mu}$ becomes a diffeomorphism. We prove the simple characterization of $\mu$ whose $H_{\mu}$ is a diffeomorphism.

Proposition 2.10 Let $\mu$ be a probability measure. Then $H_{\mu}$ is a diffeomorphism on $\mathbb{C}_{+}$if and only if $\mu=\delta_{a}$ for some $a \in \mathbb{R}$.

Proof. $\mathbb{C}_{+}$is analytically homeomorphic to the unit disc (denoted as $\Delta$ ) by the mapping $i \frac{z-i}{z+i}$. It is known that any bijective analytic map in $\Delta$ is of the form $\lambda \frac{z-b}{1+\bar{b} z}$ for some $\lambda \in \mathbb{C},|\lambda|=1$ and $b \in \mathbb{C},|b|<1$. Therefore, at least $H_{\mu}(z)$ takes the form as $\frac{a_{1} z+a_{2}}{a_{3} z+a_{4}}$, where $a_{k}$ 's are some complex numbers. Since $H_{\mu}$ is a reciprocal Cauchy transform, we have $a_{3}=0$ and $\frac{a_{1}}{a_{4}}=1$ by Proposition 2.1 in [10]. Thus $H_{\mu}(z)=z-a$ for some $a \in \mathbb{R}$. q.e.d.

\section{Atoms in Monotone Convolution}

The monotone convolution of atomic measures appears in the monotone product of matrix algebras. It is easy to prove that the monotone convolution of $m \times m$ matrix and $n \times n$ matrix becomes $m n \times m n$ matrix, which is a consequence of the algebraic construction of monotone product [14]. We study how atoms behave under monotone convolution: we prove an interlacing property of atoms in the monotone convolution of atomic measures. As a result, we obtain an interesting property which is not the case in the classical convolution (Corollary 3.3).

Theorem 3.1 (1) Let $\nu:=\sum_{k=1}^{m} \lambda_{k} \delta_{a_{k}}$ be an atomic probability measure such that $\lambda_{k}>0$, $\sum \lambda_{k}=1$ and $a_{1}<a_{2}<\cdots<a_{m}$. For any $b \in \mathbb{R}, b \neq 0, \delta_{b} \triangleright \nu$ has distinct $m$ atoms. When we write $\delta_{b} \triangleright \nu=\sum_{k=1}^{m} \mu_{k} \delta_{b_{k}}$ with $b_{1}<\cdots<b_{m}$, the atoms satisfy either $b_{1}<a_{1}<b_{2}<a_{2}<$ $\cdots<a_{m-1}<b_{m}<a_{m}$ or $a_{1}<b_{1}<a_{2}<b_{2}<\cdots<a_{m}<b_{m}$. The coefficients $\mu_{k}$ are given by $\mu_{i}=\frac{\prod_{k=1}^{m}\left(b_{i}-a_{k}\right)}{b \prod_{k \neq i}^{m}\left(b_{i}-b_{k}\right)}$.

(2) Moreover, if $b$ and $c$ are distinct real numbers, the $2 m$ atoms appearing in $\nu_{b}=\delta_{b} \triangleright \nu$ and $\nu_{c}=\delta_{c} \triangleright \nu$ are all different.

Remark 3.2 Theorem 3.1 shows a sharp difference between $\delta_{b} \triangleright \nu$ and $\delta_{b} * \nu$ : for instance, we can take $b>0$ large enough so that the atoms $\left\{b_{j}\right\}$ of $\delta_{b} * \nu$ satisfy $a_{1}<a_{2}<\cdots<a_{m}<b_{1}<$ $b_{2}<\cdots<b_{m}$, since $b_{j}=a_{j}+b$.

Corollary 3.3 Let $\mu$ be an atomic probability measure with distinct $m$ atoms and let $\nu$ be an atomic probability measure with distinct $n$ atoms. Then $\mu \triangleright \nu$ consists of exactly distinct $m n$ atoms.

Proof of Theorem. (1) The reciprocal Cauchy transform of $\delta_{b} \triangleright \nu$ is

$$
H_{\delta_{b} \triangleright \nu}(z)=\frac{\left(z-a_{1}\right) \cdots\left(z-a_{m}\right)-b \sum_{j=1}^{m} \lambda_{j} \prod_{k=1, k \neq j}^{m}\left(z-a_{k}\right)}{\sum_{j=1}^{m} \lambda_{j} \prod_{k=1, k \neq j}^{m}\left(z-a_{k}\right)} .
$$

Denote by $f(z)$ the numerator of the right hand side of (3.1). Then we have

$$
f\left(a_{1}\right)=-\lambda_{1} b\left(a_{1}-a_{2}\right)\left(a_{1}-a_{3}\right) \cdots\left(a_{1}-a_{m}\right)=(-1)^{m} b p_{1},
$$




$$
\begin{gathered}
f\left(a_{2}\right)=-\lambda_{2} b\left(a_{2}-a_{1}\right)\left(a_{2}-a_{3}\right) \cdots\left(a_{2}-a_{m}\right)=(-1)^{m-1} b p_{2}, \\
\vdots \\
f\left(a_{m}\right)=-\lambda_{m} b\left(a_{m}-a_{1}\right)\left(a_{m}-a_{2}\right) \cdots\left(a_{m}-a_{m-1}\right)=-b p_{m},
\end{gathered}
$$

where $p_{k}$ 's are some positive real numbers. The changes of signs of $f(z)$ and the behavior of $f(z)$ at $\infty$ and $-\infty$ show that there exist $m$ distinct real roots $b_{1}<\cdots<b_{m}$ of $f(z)$ as follows:

(a) $b>0 \Longrightarrow b_{k} \in\left(a_{k}, a_{k+1}\right)$ for $1 \leq k \leq m-1$, and $b_{m} \in\left(a_{m}, \infty\right)$.

(b) $b<0 \Longrightarrow b_{1} \in\left(-\infty, a_{1}\right)$ and $b_{k} \in\left(a_{k-1}, a_{k}\right)$ for $2 \leq k \leq m$.

For the denominator, we look for $\mu_{k}$ 's such that the following identity holds:

$$
\sum_{j=1}^{m} \lambda_{j} \prod_{k=1, k \neq j}^{m}\left(z-a_{k}\right)=\sum_{j=1}^{m} \mu_{j} \prod_{k=1, k \neq j}^{m}\left(z-b_{k}\right) .
$$

These $\mu_{k}$ 's are obtained as follows. When $z=b_{i},(3.2)$ becomes

$$
\mu_{i}=\frac{\sum_{j=1}^{m} \lambda_{j} \prod_{k=1, k \neq j}^{m}\left(b_{i}-a_{k}\right)}{\prod_{k \neq i}^{m}\left(b_{i}-b_{k}\right)} .
$$

Conversely, if we define the $\mu_{k}$ 's as above, the equality (3.2) holds at the different $m$ points $z=b_{k}, 1 \leq k \leq m$. Then the equality (3.2) holds identically since both sides of (3.2) are polynomials of at most degree $m-1$. Thus we have obtained

$$
H_{\delta_{b} \triangleright \nu}(z)=\frac{\prod_{k=1}^{m}\left(z-b_{k}\right)}{\sum_{k=1}^{m} \mu_{k} \prod_{j \neq k, j=1}^{m}\left(z-b_{j}\right)} .
$$

Since $f(z)$ is the numerator of $H_{\delta_{b} \triangleright \nu}(z)$, it holds that $\left(b_{i}-a_{1}\right) \cdots\left(b_{i}-a_{m}\right)=b \sum_{j=1}^{m} \lambda_{j} \prod_{k=1, k \neq j}^{m}\left(b_{i}-\right.$ $a_{k}$ ) for each $1 \leq i \leq m$. Therefore, we obtain

$$
\mu_{i}=\frac{\prod_{k=1}^{m}\left(b_{i}-a_{k}\right)}{b \prod_{k \neq i}^{m}\left(b_{i}-b_{k}\right)} .
$$

Then we obtain $\delta_{b} \triangleright \nu=\sum_{k=1}^{m} \mu_{k} \delta_{b_{k}}$.

(2) If $b$ or $c$ is equal to 0 , the claim is obvious from (1). Hereafter, we consider the case $b \neq 0$ and $c \neq 0$. In addition to $f(z)$ used in the proof of (1), we define $g(z)$ by

$$
g(z)=\left(z-a_{1}\right) \cdots\left(z-a_{m}\right)-c \sum_{j=1}^{m} \lambda_{j} \prod_{k=1, k \neq j}^{m}\left(z-a_{k}\right) .
$$

Assume that there is some $\alpha$ which satisfies both $f(\alpha)=0$ and $g(\alpha)=0$. Calculation of $f(\alpha)-g(\alpha)=0$ leads to

$$
\sum_{j=1}^{m} \lambda_{j} \prod_{k=1, k \neq j}^{m}\left(\alpha-a_{k}\right)=0
$$


where $b \neq c$ has been used. Substituting (3.7) into the expression of $f(\alpha)=0$, we have

$$
\left(\alpha-a_{1}\right) \cdots\left(\alpha-a_{m}\right)=0,
$$

which contradicts the fact that $\alpha$ is different from $a_{k}$ 's. q.e.d.

We can characterize atomic probability measures in terms of the integral representation of reciprocal Cauchy transforms by a similar argument.

Proposition 3.4 A probability measure $\nu$ has the form $\sum_{k=1}^{m} \lambda_{k} \delta_{a_{k}}$ with $a_{k}<a_{k+1}, \lambda_{k}>0$ for all $k$ if and only if its reciprocal Cauchy transform $H_{\nu}$ is of the form

$$
H_{\nu}(z)=\alpha+z+\sum_{k=1}^{m-1} \frac{\beta_{k}}{b_{k}-z},
$$

with $\beta_{k}>0$ and $\alpha \in \mathbb{R}$. Moreover, if $b_{i}{ }^{\prime} s$ are ordered as $b_{1}<b_{2}<\cdots<b_{m-1}$ then it holds that $a_{1}<b_{1}<a_{2}<b_{2}<\cdots<b_{m-1}<a_{m}$.

For an atomic probability measure $\nu$ containing more than one atom, the number of atoms in $\nu^{\triangleright n}$ increases as $n$ increases by Corollary 3.3. If we could prove that an $n$-th root of an atomic measure is again an atomic measure, then we could show that an atomic measure with finite atoms more than one is not monotone infinitely divisible by Corollary 3.3. We prove this fact next in a more general form without a reference to an $n$-th root.

We say an atom $a$ in a probability measure $\mu$ is isolated if $a \notin \overline{\operatorname{supp} \mu \backslash\{a\}}$.

Theorem 3.5 If a $\triangleright$-infinitely divisible distribution $\nu$ contains an isolated atom at $a, \nu$ is of the form $\nu=\nu(\{a\}) \delta_{a}+\nu_{a c}$, where $\nu_{a c}$ is absolutely continuous w.r.t. the Lebesgue measure and $a \notin$ supp $\nu_{a c}$. Moreover, we have

$$
\left\{u \in \operatorname{supp} \nu \backslash\{a\} ; \limsup _{v \searrow 0}\left|G_{\nu}(u+i v)\right|=\infty\right\}=\emptyset .
$$

We need the following well-known fact, which is a consequence of the theorem of de la Vallée Poussin [17].

Lemma 3.6 For a positive finite measure $\nu$, the singular part $\nu_{\text {sing }}$ is supported on $\{u \in$ supp $\left.\nu ;\left|G_{\nu}(u+i 0)\right|=\infty\right\}$.

Proof of Theorem. The probability measure $\nu$ is of the form $\nu=\lambda \delta_{a}+\mu$, where $\lambda:=$ $\nu(\{a\})>0, \mu$ is a positive finite measure and $a \notin \operatorname{supp} \mu$. It is enough to prove that $\left\{u \in \operatorname{supp} \nu \backslash\{a\} ; \lim \sup _{v \backslash 0}\left|G_{\nu}(u+i v)\right|=\infty\right\}=\emptyset$ by Lemma 3.6. We prove by reductio ad absurdum. Assume that there exists a point $a_{1} \operatorname{such}$ that $\lim \sup _{v} \backslash 0\left|G_{\nu_{a c}}\left(a_{1}+i v\right)\right|=\infty$, which implies

$$
\limsup _{v \searrow 0} H_{\nu}\left(a_{1}+i v\right)=0 .
$$

It suffices to prove that $H_{\nu}$ is not injective on $\mathbb{C}_{+}$according to $\left(c^{\prime}\right)$ explained in Section 2 . The reciprocal Cauchy transform of $\nu$ is given by

$$
\begin{aligned}
H_{\nu}(z) & =\frac{1}{\frac{\lambda}{z-a}+G_{\mu}(z)} \\
& =\frac{z-a}{\lambda+(z-a) G_{\mu}(z)} .
\end{aligned}
$$


By the assumption $a \notin \operatorname{supp} \mu, G_{\mu}$ is analytic in some small neighborhood of $a$.

Let $z_{1}$ be an arbitrary point in $\mathbb{C}_{+}$and let $f(z)$ and $g(z)$ be analytic functions defined by

$$
\begin{gathered}
f(z):=(z-a)-H_{\nu}\left(z_{1}\right)\left\{\lambda+(z-a) G_{\mu}(z)\right\} \\
g(z):=(z-a) .
\end{gathered}
$$

We note that $f\left(z_{2}\right)=0$ implies $H_{\nu}\left(z_{1}\right)=H_{\nu}\left(z_{2}\right)$. We shall prove that there exist a point $z_{1} \in \mathbb{C}_{+}$and some small open disc $D$ around $a_{2}$ such that $|f(z)-g(z)|<|f(z)|$ on $\partial D$.

We define $\eta:=\frac{1}{2} d(a, \operatorname{supp} \mu)$ and $D:=\{z \in \mathbb{C} ;|z-a|<\eta\}$, where $d(a, \operatorname{supp} \mu)$ is the distance between $a$ and $\operatorname{supp} \mu$. Then $g(z)$ has just one zero point $a$ in $D$ and $D$ does not contain $z_{1}$ if $z_{1}$ is near to $a_{1}$. We have for $z \in \partial D$

$$
|f(z)-g(z)| \leq M\left|H_{\nu}\left(z_{1}\right)\right|
$$

where $M$ is a constant independent of $z_{1}$. We also have for $z \in \partial D$

$$
\begin{aligned}
|f(z)| & \geq|z-a|-M\left|H_{\nu}\left(z_{1}\right)\right| \\
& \geq \frac{1}{2} \eta-M\left|H_{\nu}\left(z_{1}\right)\right| .
\end{aligned}
$$

If we take $z_{1}=a_{1}+y i$ with $y>0$ to satisfy $M\left|H_{\nu}\left(z_{1}\right)\right|<\frac{1}{4} \eta$, then we have $|f(z)-g(z)|<|f(z)|$ on $\partial D$. Since $g(z)$ has only one zero point $a \in D, f(z)$ also has just one zero point $z_{2}$ in $D$ by Rouche's theorem. Then it follows that $H_{\nu}\left(z_{1}\right)=H_{\nu}\left(z_{2}\right)$ and $z_{1} \neq z_{2} . \operatorname{Im} z_{2}$ might be considered to be negative, which is, however, never the case. In fact, the reciprocal Cauchy transform $H_{\nu}$ defined on $\mathbb{C} \backslash \operatorname{supp} \mu$ maps $\mathbb{C}_{+}$to $\mathbb{C}_{+}$and $\mathbb{C}_{-}$to $\mathbb{C}_{-}$. Therefore, $\operatorname{Im} z_{2}>0$, and the proof has been finished. q.e.d.

Remark 3.7 There are $\triangleright$-infinitely divisible probability distributions which contain one Dirac measure. For instance, a Dirac measure itself and the deformed arcsine law with parameter $c \geq 0$ [14] (see also Section 5 of the present paper): $d \mu_{t}=d \mu_{t, a c}+d \mu_{t, \text { sing }}$, where

$$
\begin{aligned}
& d \mu_{t, a c}(x)=\frac{1}{\pi} \frac{\sqrt{2 t-(x-c)^{2}}}{c^{2}+2 t-(x-c)^{2}} 1_{(c-\sqrt{2 t}, c+\sqrt{2 t})}(x) d x, \\
& \mu_{t, \text { sing }}=\frac{|c|}{\sqrt{c^{2}+2 t}} \delta_{c-\sqrt{c^{2}+2 t}} .
\end{aligned}
$$

Example 3.8 Let $0<\lambda<1$. The following examples do not satisfy (3.8).

(1) $\nu(d x)=\lambda \delta_{a}(d x)+\frac{1-\lambda}{c-b} 1_{(b, c)}(x) d x$ with $a \notin(b, c)$ does not satisfy (3.8), since $G_{\nu_{a c}}(z)=$ $\frac{1-\lambda}{c-b} \log \left(\frac{z-b}{z-c}\right)$.

(2) $\nu(d x)=\lambda \delta_{a}(d x)+\frac{1-\lambda}{\pi \sqrt{2-x^{2}}} 1_{(-\sqrt{2}, \sqrt{2})}(x) d x$ with $a \notin(-\sqrt{2}, \sqrt{2})$ does not satisfy (3.8) since $G_{\nu_{a c}}(z)=\frac{1-\lambda}{\sqrt{z^{2}-2}}$.

More generally, we can prove under some restrictions that a point $u$ at which the density function is not continuous satisfies $\left|G_{\nu_{a c}}(u+i 0)\right|=\infty$. We note that the deformed arcsine law $c \geq 0$ in (3.13) has an atom if and only if $c>0$, and the absolutely continuous part is a continuous function on $\mathbb{R}$ if and only if $c>0$; there are no contradictions. 


\section{Strictly Stable Distributions}

Let $b \in \mathbb{C}, c \in \mathbb{C}$ and $\alpha \in \mathbb{R}$ be constants such that $\alpha \neq 0$. We consider a $\triangleright$-infinitely divisible probability distribution $\mu^{(\alpha, b, c)}$ by the associated vector field

$$
A^{(\alpha, b, c)}(z):=\frac{b}{\alpha}(z-c)^{1-\alpha},
$$

where $z^{s}$ is defined by $z^{s}=\exp (s \log z)$ for $z \in \mathbb{C} \backslash\{x \in \mathbb{R} ; x \geq 0\}$. The range of the angle of $z$ is chosen to be $0<\arg z<2 \pi$. (Of course the factor $\frac{b}{\alpha}$ can be replaced by merely $b$; however, we use this notation since (4.2) becomes rather simple.) In order that $A^{(\alpha, b, c)}$ becomes the associated vector field to a $\triangleright$-infinitely divisible distribution, the following conditions are necessary and sufficient:

(a) $A^{(\alpha, b, c)}$ maps $\mathbb{C}_{+}$into $\mathbb{C}_{+} \cup \mathbb{R}$;

(b) $\lim _{y \rightarrow \infty} \frac{\operatorname{Im} A^{(\alpha, b, c)}(x+i y)}{y}=0$ for some $x$.

By careful observation upon the motion of angles, we can see that $A^{(\alpha, b, c)}$ satisfies (a) and (b) if and only if

(1) $\operatorname{Im} c \leq 0$,

(2) $0<\alpha \leq 2$,

(3) $0 \leq \arg b \leq \alpha \pi$ for $0<\alpha \leq 1$ and $(\alpha-1) \pi \leq \arg b \leq \pi$ for $1<\alpha \leq 2$,

except for the case $\alpha=1$. If $\alpha=1, A^{(1, b, c)}$ does not depend on $c$ and the condition (1) is not needed. We can write explicitly the corresponding reciprocal Cauchy transform:

$$
H^{(\alpha, b, c)}(z)=c+\left\{(z-c)^{\alpha}+b\right\}^{\frac{1}{\alpha}} .
$$

When we consider the corresponding convolution semigroup $\left\{\mu_{t}^{(\alpha, b, c)}\right\}_{t \geq 0}$, the formula (4.2) becomes

$$
H_{t}^{(\alpha, b, c)}(z)=c+\left\{(z-c)^{\alpha}+b t\right\}^{\frac{1}{\alpha}} .
$$

This family is an extension of deformed arcsine laws in [14] $(\operatorname{Im} c=0, \alpha=2)$, Cauchy distributions $(\alpha=1, b=\beta i$ with $\beta>0)$ and delta measures $(\alpha=1, \operatorname{Im} b=0)$. Moreover, this family gives good examples when we study support properties of general $\triangleright$-infinitely divisible distributions [9].

We show that the family $\left\{\mu^{(\alpha, b, 0)}\right\}$ gives all strictly monotone stable distributions which we define now. Let $D_{\lambda}$ be the dilation operator defined by

$$
D_{\lambda} \mu(B)=\mu\left(\lambda^{-1} B\right),
$$

where $B$ is an arbitrary Borel set and $\mu$ is an arbitrary Borel measure.

Definition 4.1 Let $\mu$ be a $\triangleright$-infinitely divisible distribution. Then there exists a unique weakly continuous $\triangleright$-convolution semigroup $\left\{\mu_{t}\right\}_{t \geq 0}$ such that $\mu_{1}=\mu$ and $\mu_{0}=\delta_{0} . \mu$ is called a strictly $\triangleright$-stable distribution if for any $a>0$ there exists $b(a)>0$ such that

$$
\mu_{a}=D_{b(a)} \mu .
$$


(4.5) is equivalent to the following equality:

$$
H_{\mu_{a}}(z)=b(a) H_{\mu}\left(b(a)^{-1} z\right) \text { for all } z \in \mathbb{C}_{+} .
$$

We often write $H_{t}=H_{\mu_{t}}$ for simplicity.

Remark 4.2 We do not treat unbounded operators which will be interesting in the study of strictly $\triangleright$-stable distributions; we deal with only probability distributions.

Lemma 4.3 Assume that $\mu$ is a strictly $\triangleright$-stable distribution with $\mu \neq \delta_{0}$.

(1) $b(a)$ is unique for each $a>0$ and $b(a)$ is a continuous function of $a$.

(2) It holds that $H_{a t}(z)=b(a) H_{t}\left(b(a)^{-1} z\right)$ for all $a>0, t \geq 0$ and $z \in \mathbb{C}_{+}$.

(3) There exists some $h \in \mathbb{R}$ such that $b(a)=a^{h}$ for all $a>0, t \geq 0$.

Proof. (1) The proof of the uniqueness of $b(a)$ given below is almost the same as in Lemma 13.7 in [18]. Fix $a>0$. Assume that there exist $b>b^{\prime}$ such that $H_{a}(z)=b H_{1}\left(b^{-1} z\right)=b^{\prime} H_{1}\left(b^{\prime-1} z\right)$ for all $z \in \mathbb{C}_{+}$. Then we have $\mu\left(\frac{d x}{b}\right)=\mu\left(\frac{d x}{b^{\prime}}\right)$, and hence, we get $\mu\left(c^{n} d x\right)=\mu(d x)$ with $c=\frac{b}{b^{\prime}}>1$ for all $n \in \mathbb{N}$. Letting $n \rightarrow \infty$, we have $\mu=\delta_{0}$, which is a contradiction. Then we have the uniqueness of $b$. The proof of the continuity of $b(a)$ is the same as Lemma 13.9 in [18] and we omit the proof.

(2) We fix $a>0$. Define two families of probability measures $\nu_{t}:=\mu_{a t}(d x)$ and $\lambda_{t}:=\mu_{t}\left(\frac{d x}{b(a)}\right)$. Since $\mu$ is strictly $\triangleright$-stable, we have $\nu_{1}=\lambda_{1}$. Moreover, both $\left\{\nu_{t}\right\}$ and $\left\{\lambda_{t}\right\}$ constitute monotone convolution semigroups. Therefore, we obtain $\nu_{t}=\lambda_{t}$ for all $t \geq 0$ by the uniqueness result obtained in [3].

(3) By the result (2) it holds that

$$
H_{a a^{\prime} t}(z)=b(a) H_{a^{\prime} t}\left(b(a)^{-1} z\right)=b(a) b\left(a^{\prime}\right) H_{t}\left(b(a)^{-1} b\left(a^{\prime}\right)^{-1} z\right)
$$

for all $a, a^{\prime}>0$ and $t \geq 0$. Therefore, by (1) we have

$$
b\left(a a^{\prime}\right)=b(a) b\left(a^{\prime}\right)
$$

for all $a, a^{\prime}>0$. It is a well known fact that a continuous function satisfying the equation (4.8) is of the form $b(a)=a^{h}$. q.e.d.

Definition 4.4 The reciprocal of $h$ in Lemma 4.3 is called the index of $\mu$. We denote the index by $\alpha$ and in this case we call $\mu$ a strictly $\triangleright$ - $\alpha$-stable distribution.

Assume that $\mu$ is a $\triangleright$-infinitely divisible distribution. Let $A$ be the associated vector field in (1.5). The following equivalent conditions are useful in the classification of strictly $\triangleright$-stable distributions:

(1) $\mu$ is a strictly $\triangleright$ - $\alpha$-stable distribution;

(2) $H_{a t}(z)=a^{\frac{1}{\alpha}} H_{t}\left(a^{-\frac{1}{\alpha}} z\right)$ for all $z \in \mathbb{C}_{+}$;

(3) $A(z)=a^{\frac{1}{\alpha}-1} A\left(a^{-\frac{1}{\alpha}} z\right)$ for all $z \in \mathbb{C}_{+}$. 
Theorem 4.5 Assume that $\mu$ is a strictly $\triangleright$-stable distribution with $\mu \neq \delta_{0}$. Then the index $\alpha$ of $\mu$ satisfies $0<\alpha \leq 2$. Moreover, there exists $b \in \mathbb{C}$ such that $\mu=\mu^{(\alpha, b, 0)}$, where $b$ satisfies the following conditions:

$$
\begin{aligned}
& 0 \leq \arg b \leq \alpha \pi \text { if } 0<\alpha \leq 1, \\
& \cdot(\alpha-1) \pi \leq \arg b \leq \pi \text { if } 1<\alpha \leq 2 .
\end{aligned}
$$

Proof. We have the equation $A(z)=a^{\frac{1}{\alpha}-1} A\left(a^{-\frac{1}{\alpha}} z\right)$ for all $a>0$ and $z \in \mathbb{C}_{+}$since $\mu$ is a strictly $\triangleright$ - $\alpha$-stable distribution. Differentiating the equation w.r.t. $a$, we obtain $A^{\prime}\left(a^{-\frac{1}{\alpha}} z\right)=$ $\frac{1-\alpha}{a^{-\frac{1}{\alpha}} z} A\left(a^{-\frac{1}{\alpha}} z\right)$. Putting $a=1$, we obtain

$$
A^{\prime}(z)=\frac{1-\alpha}{z} A(z)
$$

It follows from the differential equation (4.9) that

$$
A(z)=\frac{b}{\alpha} z^{1-\alpha}
$$

for some constant $b \in \mathbb{C}$. As explained in the beginning of this section, we obtain the conclusion. q.e.d.

Remark 4.6 (1) The characterization of strictly $\triangleright$-stable distributions in terms of the associated vector field $A(z)=b z^{1-\alpha}$ is very similar to the cases of free [5] and boolean [19].

(2) Cauchy distributions are strictly $\triangleright-1$-stable distributions, which is also the case in classical, free and boolean cases.

\section{$5 \quad$ Examples}

We consider the family of distributions $\mu_{t}^{(\alpha, b, c)}$ introduced in (4.3) under the following restrictions: $b=1$ when $0<\alpha<1$ and $b=-1$ when $1 \leq \alpha \leq 2$. In terms of the reciprocal Cauchy transform, we have

$$
H_{t}^{(\alpha, 1, c)}(z)=c+\left\{(z-c)^{\alpha}+t\right\}^{\frac{1}{\alpha}} \text { for } 0<\alpha<1
$$

and

$$
H_{t}^{(\alpha,-1, c)}(z)=c+\left\{(z-c)^{\alpha}-t\right\}^{\frac{1}{\alpha}} \text { for } 1 \leq \alpha \leq 2 .
$$

We write simply as $H_{t}(z)$ and $\mu_{t}$ when there are no confusions. The support of an absolutely continuous part and the positions of atoms for various parameters $\alpha, c, t(t>0)$ are summarized as follows:

(1) $\operatorname{Im} c<0$

$$
\begin{aligned}
& \mu_{t}=\mu_{t, a c}, \\
& \operatorname{supp} \mu_{t, a c}=\mathbb{R} .
\end{aligned}
$$


(2) $\operatorname{Im} c=0, \operatorname{Re} c \geq 0, \alpha=2$

$$
\begin{aligned}
& \mu_{t}=\mu_{t, a c}+\mu_{t, \text { sing }}, \\
& \operatorname{supp} \mu_{t, a c}=[c-\sqrt{t}, c+\sqrt{t}], \\
& \mu_{t, \text { sing }}=\frac{|c|}{c^{2}+t} \delta_{c-\sqrt{c^{2}+t}} .
\end{aligned}
$$

(3) $\operatorname{Im} c=0, \operatorname{Re} c<0, \alpha=2$

$$
\begin{aligned}
& \mu_{t}=\mu_{t, a c}+\mu_{t, \text { sing }}, \\
& \operatorname{supp} \mu_{t, a c}=[c-\sqrt{t}, c+\sqrt{t}], \\
& \mu_{t, \text { sing }}=\frac{|c|}{c^{2}+t} \delta_{c+\sqrt{c^{2}+t}} .
\end{aligned}
$$

(4) $\operatorname{Im} c=0, \operatorname{Re} c \geq 0,1<\alpha<2$

$$
\begin{aligned}
& \mu_{t}=\mu_{t, a c}, \\
& \operatorname{supp} \mu_{t, a c}=\left(-\infty, c+t^{\frac{1}{\alpha}}\right] .
\end{aligned}
$$

(5) $\operatorname{Im} c=0, \operatorname{Re} c<0,1<\alpha<2$

$$
\begin{aligned}
& \mu_{t}=\mu_{t, a c}+\mu_{t, s i n g}, \\
& \operatorname{supp} \mu_{t, a c}=\left(-\infty, c+t^{\frac{1}{\alpha}}\right], \\
& \mu_{t, \text { sing }}=\left(\frac{|c|^{\alpha}}{|c|^{\alpha}+t}\right)^{\frac{\alpha-1}{\alpha}} \delta_{c+\left(|c|^{\alpha}+t\right)^{\frac{1}{\alpha}}}
\end{aligned}
$$

(6) $\operatorname{Im} c=0, \operatorname{Re} c \geq 0,0<\alpha<1$

$$
\begin{aligned}
& \mu_{t}=\mu_{t, a c}, \\
& \operatorname{supp} \mu_{t, a c}=(-\infty, c] .
\end{aligned}
$$

(7) $\operatorname{Im} c=0, \operatorname{Re} c<0,0<\alpha<1$

$$
\begin{aligned}
& \mu_{t}=\mu_{t, a c}+\mu_{t, s i n g}, \\
& \operatorname{supp} \mu_{t, a c}=(-\infty, c], \\
& \mu_{t, \text { sing }}= \begin{cases}\left(\frac{|c|^{\alpha}-t}{|c|^{\alpha}}\right)^{\frac{1-\alpha}{\alpha}} \delta_{c+\left(|c|^{\alpha}-t\right)^{\frac{1}{\alpha}},} & 0 \leq t<|c|^{\alpha}, \\
0, & t \geq|c|^{\alpha} .\end{cases}
\end{aligned}
$$

(8) $\operatorname{Im} c=0, \alpha=1$

$$
\mu_{t, s i n g}=\delta_{t c} .
$$

Several features can be seen from the above examples. In (6), the support of the absolutely continuous part does not vary as a function of $t$; however, it varies as a function of $t$ in (5), for instance. One can see in (7) that there exists a probability measure $\mu$ which contains a delta 
measure although $\mu \triangleright \mu$ does not contain a delta measure. Therefore, we can conclude that monotone convolution does not conserve the absolute continuity of probability distributions.

We calculate $\mu_{t}$ explicitly in the case of $\alpha=2$ and $\operatorname{Im} c=0$, and in the case of $\alpha=\frac{1}{2}$ and $\operatorname{Im} c=0$. When $\alpha=2$ and $\operatorname{Im} c=0$, the result is shown in [14]. Coefficients of delta measures are, however, not shown in [14]. It is important from the viewpoint of Theorem 3.5 to check that two delta measures do not appear at the same time.

1. $\alpha=2$ and $\operatorname{Im} c=0$ The Cauchy transform is given by

$$
G_{t}(z)=\left[c+\left|(x-c)^{2}-t-y^{2}+2 i y(x-c)\right|^{\frac{1}{2}} \exp \left\{\frac{i}{2} \arg \left((x-c)^{2}-t-y^{2}+2 i y(x-c)\right)\right\}\right]^{-1} .
$$

case 1: $|x-c|>\sqrt{t}$

Since $(x-c)^{2}-t>0$, we have

$$
\lim _{y \rightarrow+0} \exp \left\{\frac{i}{2} \arg \left((x-c)^{2}-t-y^{2}+2 i y(x-c)\right)\right\}= \begin{cases}1, & x-c>+\sqrt{t} \\ -1, & x-c<-\sqrt{t}\end{cases}
$$

Therefore, we have the limit

$$
\lim _{y \rightarrow+0} G_{t}(x+i y)= \begin{cases}\frac{1}{c+\sqrt{(x-c)^{2}-t}}, & x-c>+\sqrt{t} \\ \frac{1}{c-\sqrt{(x-c)^{2}-t}}, & x-c<-\sqrt{t} .\end{cases}
$$

case 2: $|x-c| \leq \sqrt{t}$

In this case we have

$$
\lim _{y \rightarrow+0} \exp \left\{\frac{i}{2} \arg \left((x-c)^{2}-t-y^{2}+2 i y(x-c)\right)\right\}=i .
$$

Hence we obtain

$$
\begin{aligned}
\lim _{y \rightarrow+0} G_{t}(x+i y) & =\frac{1}{c+i \sqrt{t-(x-c)^{2}}} \\
& =\frac{c-i \sqrt{t-(x-c)^{2}}}{c^{2}+t-(x-c)^{2}}
\end{aligned}
$$

The absolutely continuous part of $\mu_{t}$ is

$$
d \mu_{t, a c}(x)=\frac{1}{\pi} \frac{\sqrt{t-(x-c)^{2}}}{c^{2}+t-(x-c)^{2}} 1_{(c-\sqrt{t}, c+\sqrt{t})}(x) d x .
$$

There is a delta measure at $a$ only when $\lim _{y \backslash 0} i y G_{t}(a+i y)>0$. As a possible position of a delta measure, there is only one point $a$ with $|x-c|>\sqrt{t}$ which satisfies

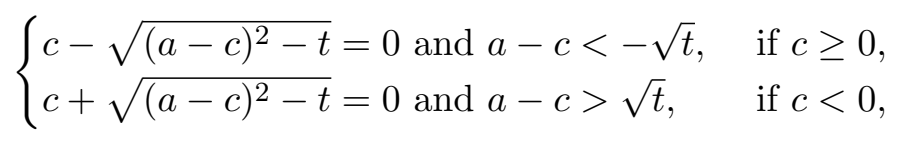


that is, $a=c-\sqrt{c^{2}+t}$ for $c \geq 0$ and $a=c+\sqrt{c^{2}+t}$ for $c<0$. Therefore, the singular part of $\mu_{t}$ is

$$
\mu_{t, s i n g}= \begin{cases}A \delta_{c-\sqrt{c^{2}+t}}, & c \geq 0, \\ B \delta_{c+\sqrt{c^{2}+t},} & c<0 .\end{cases}
$$

It is not difficult to calculate $\mu_{t, a c}(\mathbb{R})=1-\frac{|c|}{\sqrt{c^{2}+t}}$. Then one can determine the singular part completely:

$$
\mu_{t, \operatorname{sing}}= \begin{cases}\frac{|c|}{\sqrt{c^{2}+t}} \delta_{c-\sqrt{c^{2}+t}}, & c \geq 0 \\ \frac{|c|}{\sqrt{c^{2}+t}} \delta_{c+\sqrt{c^{2}+t}}, & c<0 .\end{cases}
$$

2. $\alpha=\frac{1}{2}$ and $\operatorname{Im} c=0$ The reciprocal Cauchy transform is given by

$$
\begin{aligned}
H_{t}(z) & =c+\left\{(z-c)^{\frac{1}{2}}+t\right\}^{2} \\
& =t^{2}+z+2 t \sqrt{z-c} .
\end{aligned}
$$

Here the branch of $(z-c)^{\frac{1}{2}}$ is taken such that $\sqrt{-1}=i$. If $x-c>0$, then

$$
G_{t}(x+i 0)=\frac{1}{t^{2}+x+2 t \sqrt{x-c}} .
$$

If $x-c \leq 0$, then

$$
\begin{aligned}
G_{t}(x+i 0) & =\frac{1}{t^{2}+x+2 t i \sqrt{c-x}} \\
& =\frac{t^{2}+x-2 t i \sqrt{c-x}}{\left(t^{2}+x\right)^{2}+4 t^{2}(c-x)} .
\end{aligned}
$$

To know the position and the weight of a delta measure it is important to calculate the following quantity:

$$
\lim _{y \rightarrow+0} y G_{t}(a+i y)= \begin{cases}\frac{\sqrt{|c|}-t}{i \sqrt{|c|}}, & \text { if } a=t^{2}-2 t \sqrt{|c|}, c<0, t \leq \sqrt{|c|}, \\ 0, & \text { otherwise. }\end{cases}
$$

If $c<0$, there is a delta measure in this distribution at $x=t^{2}-2 t \sqrt{|c|}$ and it disappears at the time $t=\sqrt{|c|}$. The maximum $b\left(\mu_{t}\right)$ of the support of $\mu_{t}$ is

$$
b\left(\mu_{t}\right)= \begin{cases}t^{2}-2 t \sqrt{|c|}, & 0 \leq t \leq \sqrt{|c|} \\ c, & t \geq \sqrt{|c|} .\end{cases}
$$

$b\left(\mu_{t}\right)$ decreases as a function of $t$. The absolutely continuous part is calculated as

$$
d \mu_{t, a c}(x)=\frac{1}{\pi} \frac{2 t \sqrt{c-x}}{\left(t^{2}+x\right)^{2}+4 t^{2}(c-x)} 1_{(-\infty, c)}(x) d x .
$$

Convergence of $\mu_{t}$ as $t \rightarrow 0$ is

$$
\lim _{t \rightarrow 0} \mu_{t}= \begin{cases}\delta_{0}, & c \geq 0, \\ 0, & c<0 .\end{cases}
$$

Finally, the distribution is obtained as follows: 


$$
\mu_{t}= \begin{cases}\mu_{t, a c}, & c \geq 0,0<t<\infty, \\ \mu_{t, a c}+\frac{\sqrt{|c|}-t}{\sqrt{|c|}} \delta_{t^{2}-2 t \sqrt{|c|},} & c<0,0<t \leq \sqrt{|c|} \\ \mu_{t, a c}, & c<0, t \geq \sqrt{|c|} .\end{cases}
$$

\section{Acknowledgments}

The author expresses his great appreciation to Prof. Izumi Ojima for his warm encouragements and valuable suggestions during his master course. He is also grateful to Mr. Hiroshi Ando, Mr. Ryo Harada, Mr. Kazuya Okamura and Mr. Hayato Saigo for their interests in his research and many helpful discussions. He is also grateful to Prof. Muraki and the referee for guiding him to important references. This work was supported by the Grant-in-Aid for JSPS Research Fellows (DC).

\section{References}

[1] N. I. Akhiezer, The Classical Moment Problem (English transl.), Oliver and Boyd, 1965.

[2] F. G. Avkhadiev and L. A. Aksent'ev, The main results on sufficient conditions for an analytic function to be Schlicht, Russian Math. Surveys 30:4 (1975), 1-64.

[3] S. Belinschi, Complex analysis methods in noncommutative probability, available at arXiv:math/0602343v1.

[4] E. Berkson and H. Porta, Semigroups of analytic functions and composition operators, Michigan Math. J. 25 (1978), 101-115.

[5] H. Bercovici and D. Voiculescu, Free Convolution of Measures with Unbounded Support, Indiana Univ. Math. J. Vol. 42, No. 3 (1993), 733-773.

[6] R. Bhatia, Matrix Analysis. Springer, 1997.

[7] C. C. Cowen, Iteration and the solution of functional equations for functions analytic in the unit disk, Trans. Amer. Math. Soc. 265, no.1 (1981), 69-95.

[8] U. Franz, Monotone and boolean convolutions for non-compactly supported probability measures, to appear in Indiana Univ. Math. J.

[9] T. Hasebe, in preparation.

[10] H. Maassen, Addition of freely independent random variables, J. Funct. Anal. 106 (1992), 409-438.

[11] J. Milnor, remarks on infinite-dimensional Lie groups, pp. 1007-1057 in relativité, Groupes et Topologie II, B. DeWitt and R. Stora (Eds), North-Holland, Amsterdam, 1983.

[12] N. Muraki, Non-commutative Brownian motion in monotone Fock space, Commun. Math. Phys. 183 (1997), 557-570. 
[13] N. Muraki, Monotonic independence, monotonic central limit theorem and monotonic law of small numbers, Inf. Dim. Anal. Quantum Probab. Rel. Topics 4 (2001), 39-58.

[14] N. Muraki, Monotonic convolution and monotonic Lévy-Hinčin formula, preprint, 2000.

[15] R. Nevanlinna and V. Paatero, Introduction to complex analysis, Chelsea Publishing Company, 1969.

[16] Ch. Pommerenke, On the iteration of analytic functions in a halfplane, I, J. London Math. Soc. (2), 19, no.3 (1979), 439-447.

[17] S. Saks, Theory of the Integral, Hafner, New York, 1937.

[18] K. Sato, Lévy Processes and Infinitely Divisible Distributions, Cambridge University Press, Cambridge, 1999.

[19] R. Speicher and R. Woroudi, Boolean convolution, in Free Probability Theory, ed. D. V. Voiculescu, Fields Inst. Commun., vol. 12 (Amer. Math. Soc., 1997), 267-280.

[20] F. W. Steutel and K. Harn, Infinite Divisibility and Probability Distributions on the Real Line, Marcel Dekker, 2004. 